\title{
Learning from Nature about Principles of Hierarchical Materials
}

\author{
Huajian Gao \\ Brown University
}

\begin{abstract}
Natural materials such as bone, shell, tendon and the attachment system of gecko exhibit multi-scale hierarchical structures. Here we summarize some recent studies on an idealized self-similar hierarchical model of bone and bone-like materials, and discuss mechanical principles of self-similar hierarchy, in particular to show how the characteristic length, aspect ratio and density at each hierarchical level can be selected to achieve flaw tolerance and superior stiffness and toughness across scale.
\end{abstract}

\section{INTRODUCTION}

Multi-level structural hierarchy can be observed in many biological systems including bone [1-6] and attachment pads of gecko [7-9]. In fact, structural hierarchy is a rule of nature. Hierarchical structures can be observed in all biosystems from chromosome, protein, cell, tissue, organism, to ecosystems. What are the roles and principles of structural hierarchy? What determine the size scales and other geometrical factors in a hierarchical material? These questions should be of general interest to both engineering and biological systems.

Recent studies on biological materials have shown that the characteristic size at each level of structural hierarchy may have been selected to ensure tolerance of material/structural flaws. For example, it has been demonstrated $[10,11]$ that, due to their nanoscale characteristic size, the mineral bits in bone and bone-like materials tend to fail not by propagation of preexisting cracks but by uniform rupture at the limiting strength of the material. For biological adhesion systems [7,8,12], similar transition from crack-like failure to uniform rupture has also been discussed [13]; the adhesion strength is affected not only by the size but also by the shape of the contacting surfaces: the smaller the size, the less important the shape, and shapeinsensitive optimal adhesion was found to become possible

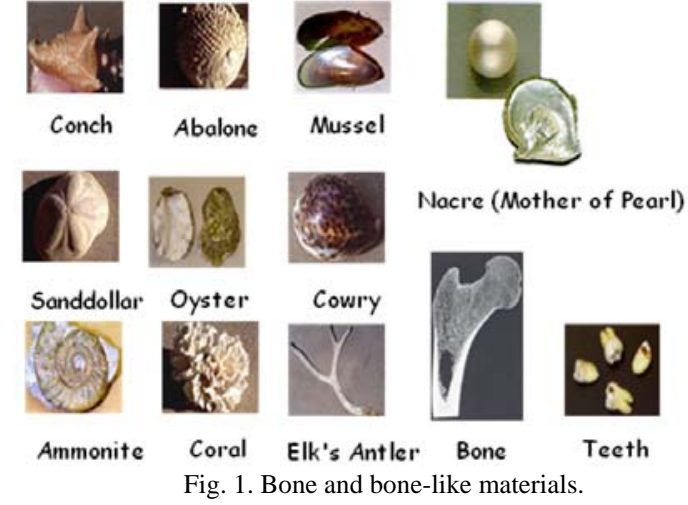

when the structural size is reduced to below a critical length around $100 \mathrm{~nm}$ for van der Waals adhesion [14].

Here we summarize some recent studies on an idealized selfsimilar hierarchical model mimicking the structure of bone [6,15]. It is known that bone and bone-like materials (Fig. 1) exhibit hierarchical structures over many length scales. For example, sea shells have 2 to 3 levels of lamellar structure [1,2,16-18], while vertebral bone has 7 levels of structural hierarchy [2, 19-22] (Fig. 2). Although the higher level structures of bone and bone-like materials show great complexity and variations, they exhibit a generic nanostructure (Fig. 2) at the most elementary level of structural hierarchy consisting of nanometer sized hard mineral crystals arranged in a parallel staggered pattern in a soft protein matrix $[3,5,10]$. The nanostructure of tooth enamel shows needle-like (15$20 \mathrm{~nm}$ thick and $1000 \mathrm{~nm}$ long) crystals embedded in a relatively small volume fraction of a soft protein matrix [2325]. The nanostructure of dentin and bone consists of plate-like (2-4nm thick and up to $100 \mathrm{~nm}$ long) crystals embedded in a
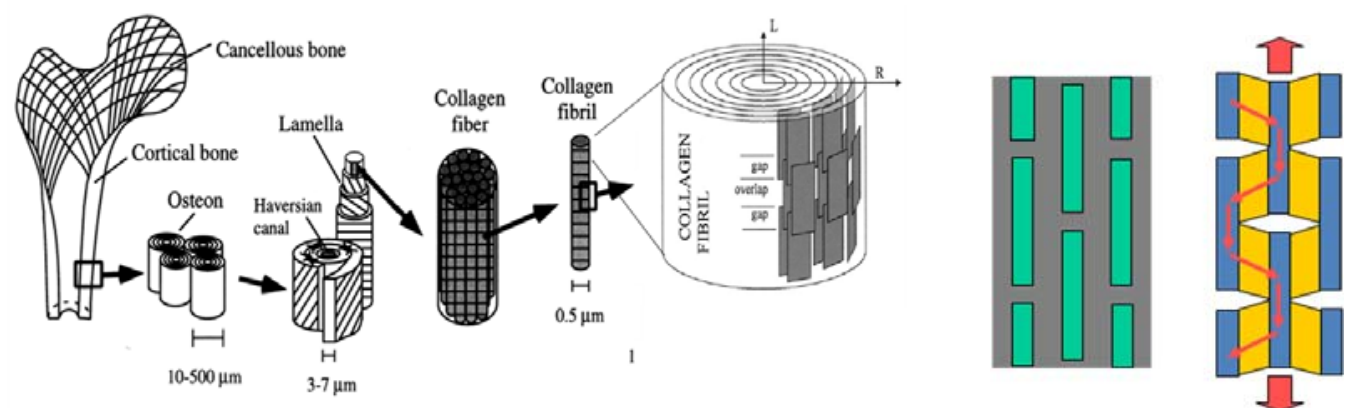

Fig. 2. Hierarchical structures of bone and the generic nanostructure of bone-like materials [3, 10, 21]. 

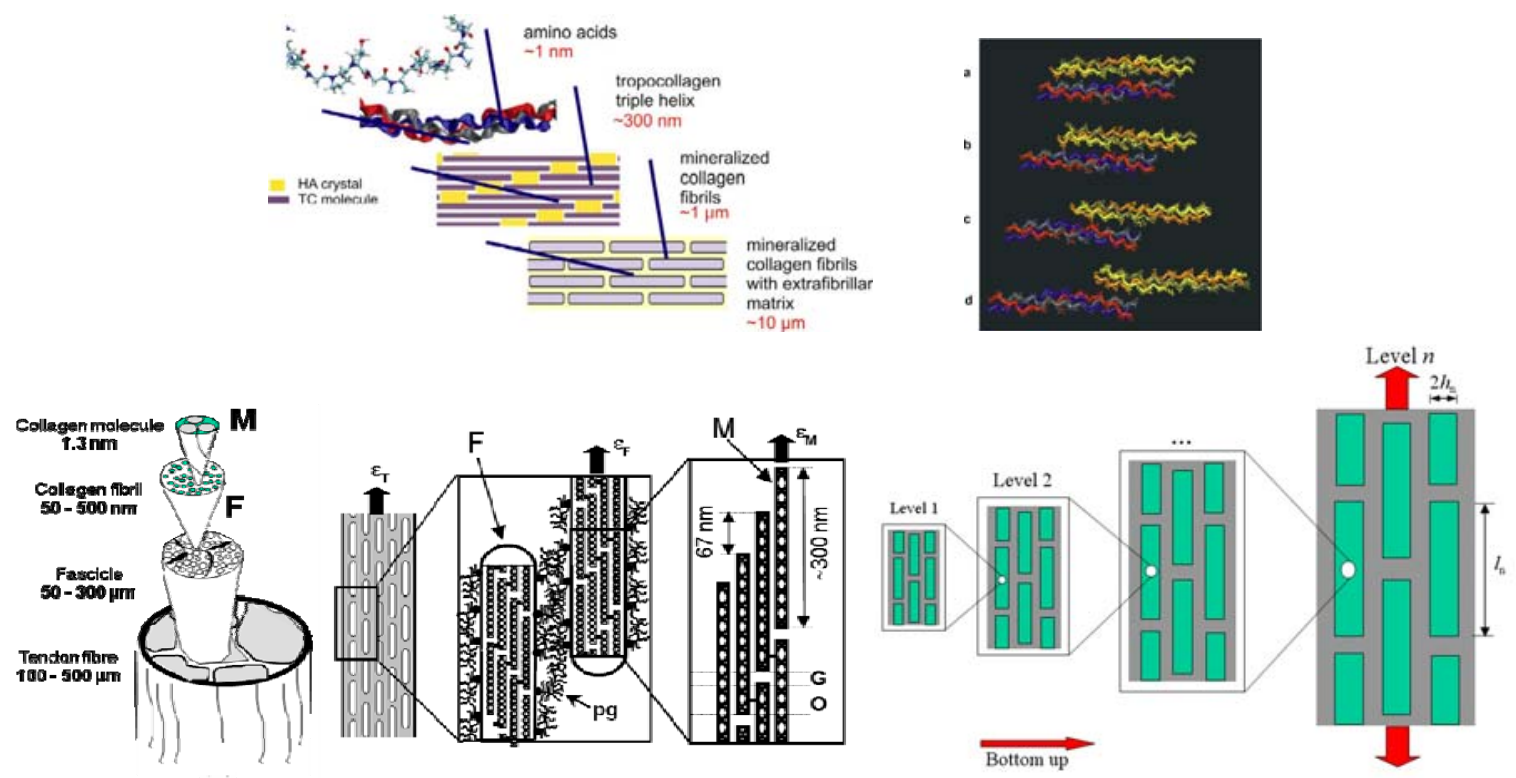

Figure 3. Self-similar structural hierarchy in bone and tendon $[6,15,27,28]$.

collagen-rich protein matrix [19,20], with the volume ratio of mineral to matrix on the order of 1 to 2 . Nacre is made of very high volume fraction of plate-like crystals (200-500nm thick and a few micrometers long) with a small amount of soft matrix in between [1,2,16-18,26]. Bone and nacre are constructed with basically the same type of nanostructure made of staggered plate-like hard inclusions in a soft matrix. This staggered nanostructure is primarily subjected to uniaxial loading, as shown in Fig. 2.

As an example of hierarchical structures, we discuss an idealized hierarchical material with multiple levels of selfsimilar structures mimicking the staggered nanostructure of bone [6,15], as shown in Fig. 3. The resulting composite structure is still made of mineral and protein at some volume fraction, but the material is now distributed in a highly nonhomogeneous way to form a hierarchical material with different properties at different length scales. In principle, this self-similar model can have arbitrary levels of hierarchy and at each level exhibits the same structure of slender hard plates arranged in a parallel staggered pattern in a soft matrix, similar to the nanostructure of bone. At each level of hierarchy, three mechanical principles will be required to determine three geometrical parameters: the width, length and volume fraction of hard inclusions.

\section{MECHANICAL PRINCIPLES OF SELF-SIMILAR HIERARCHY}

\section{PRINCIPLE I: SELECTION OF CHARACTERISTIC LENGTH FOR UNIFORM STRESS IN HARD PARTICLES AT FAILURE}

Biological systems must be robust for survival. Therefore, the first principle of biocomposite is postulated to be that of flaw tolerance. Since the staggered biocomposite structure is primarily subjected to uniaxial tension, as shown in Fig. 2, the path of load transfer in the structure follows a tension-shear chain with the hard plates under tension and the soft matrix under shear. Analysis based on this tension-shear chain model $[10,29]$ showed that the integrity of biocomposites hinges upon the tensile strength of the hard particles. In order to keep the structure intact during deformation, the hard particles must be able to sustain large tensile load without fracture, while the protein/particle interface and the protein layer transfer load via shear and dissipate energy.

The essence of flaw tolerance is to maximize the load carrying potential of the hard particles, which amounts to avoiding crack propagation until the material reaches its limiting strength. Theoretical estimation based on interatomic force laws shows that the theoretical strength is around $E$ / 30, where $E$ is the Young's modulus. In reality, however, such high strength is rarely observed due to the inevitable presence of crack-like flaws which, under external loading, induce stress concentration near the tips of these flaws. As the external load reaches a critical value, the solid would fracture via crack propagation instead of simultaneous breaking of all bonds as assumed in the definition of theoretical strength. Under this circumstance, the load carrying capacity of the material is not utilized most efficiently since only a small fraction of material is maximally stressed at any instant of time during failure, leading to a much reduced "apparent" strength in contrast to the theoretical value. From the robustness point of view, an ideal scenario is to achieve the state of flaw tolerance $[10,11]$ in which the material fails at the theoretical strength irrespective of the presence and size of cracks. Due to the random, unpredictable nature of crack-like flaws, it may seem at a first glance extremely difficult or impossible to eliminate 
stress concentration for large cracks so as to ensure uniform stress at material failure. However, theoretical arguments based on the well established concepts in fracture mechanics $[6,15]$ showed that this is actually possible with hierarchical material design, provided that the characteristic length at each hierarchical level is appropriately selected.

\section{Principle II: Selection of aspect ratio for uniform stress in the matrix at failure}

The next question is how the aspect ratio of the hard plates should be chosen. There have been two interesting considerations here. The first is to keep the stress in the matrix uniform up to failure. In a way, this is actually similar to Principle I. For this to happen, the aspect ratio of the hard particle must not exceed a critical value. This concept is similar to the shear lag model [31-33] used in modeling mechanical properties of composites [34].

The second consideration is that, even when the shear stress in the matrix is uniform, as assumed in the tension-shear chain model, the aspect ratio of the particles could be further adjusted to ensure that the matrix, the particles and their interfaces reach their respective limiting strengths at the same time $[6,10]$.

Therefore, the aspect ratio of particles can be selected to ensure (1) that the stress in the matrix remains uniform even at the failure point and (2) that the soft matrix, the hard particles and their interfaces fail at the same time.

Similar concept of a characteristic length has been developed for optimal overlap length between two tropocollagen molecules [35], where it was shown that homogeneous shear is only possible when the overlap length is below the characteristic length and that fracture like slip pulses develop for larger overlapping lengths.

\section{Principle III: Selection of volume fraction of hard particles for optimal balance between stiffness and toughness}

After the characteristic length and aspect ratio of hard particles have been determined to ensure uniform stress of materials up to the failure point in both particles and matrix, the volume fraction of hard particles can be further selected to achieve the desired objective in mechanical properties. For bone, this objective is expected to be a combined stiffness and toughness. The toughness of the structure is expected to rise as the volume fraction of the hard material decreases. That is, the more soft material in the structure, the higher the toughness, although this may occur at the cost of compromising stiffness of the structure. For example, the toughness is expected to be of increasing importance for sea shells, bone and antelope horn, which correlates with their respective mineral contents of $95 \%$, $45 \%$ and $35 \%$, respectively. We will show that the toughness of a hierarchical material at one level depends on the strength at the lower level, i.e. higher strength at the (n-1)th level results in higher toughness at the nth level. This is a unique property of hierarchical materials.

That bone and bone-like materials have been evolved to achieve a balance between toughness and stiffness should be apparent in their staggered structure. Studies based on genetic algorithms $[36,6]$ have shown that the staggered nanostructure of bone could be interpreted as an evolutionary result with the objective to simultaneously optimize stiffness and toughness.

For the self-similar structural shown in Fig. 3, the above three principles can then be adopted to determine the structure at each level: the characteristic size, the aspect ratio and the volume fraction of hard particles. The first principle is to select the characteristic size of the structure to achieve uniform stress in the hard particles at failure, so as to ensure random cracklike flaws in the material/structure will not propagate until the entire structure fails near its theoretical strength. In other words, Principle I is to ensure that the stress distribution in the hard particles remain uniform so that the maximum load carrying potential of the hard material can be realized. The second principle is to select the aspect ratio of the hard particles to ensure that the shear stress in the matrix material is uniform so that the maximum load transferring capacity of the soft matrix is realized. Also, even if the shear stress in the matrix is uniform, the aspect ratio may need to be further checked to make sure that the particles do not fail before the matrix. Once the potential of both hard and soft material is realized to the maximum extent, the third principle is to select the volume fraction of hard material (mineral) to achieve the desired balance between stiffness and toughness. Generally, higher mineral content promotes stiffness, while higher amount of protein promotes toughness.

* Huajian Gao, Division of Engineering, Brown University, Providence, RI 02912, USA. Email: huajian_gao@brown.edu

\section{References}

1. Currey, J.D., 1977. Mechanical properties of mother of pearl in tension. Proc. R. Soc. Lond. B 196, 443-463.

2. Currey, J.D., 1984. The Mechanical Adaptations of Bones. Princeton University Press, Princeton, NJ, pp. 24-37.

3. Jäger, I., Fratzl, P., 2000. Mineralized collagen fibrils: a mechanical model with a staggered arrangement of mineral particles. Biophys. J. 79, 1737-1746.

4. Fratzl, P., Burgert, I., Gupta, H.S., 2004a. On the role of interface polymers for the mechanics of natural polymeric composites. Phys. Chem. Chem. Phys. 6, 5575-5579.

5. Fratzl, P., Gupta, H.S., Paschalis, E.P., Roschger, P., 2004b. Structure and mechanical quality of the collagen-mineral nano-composite in bone. J. Mater. Chem. 14, 2115-2123.

6. Gao, H., 2006. Application of fracture mechanics concepts to hierarchical biomechanics of bone and bon-like materials. Int. J. Fracture138, 101137.

7. Autumn, K., Liang, Y.A., Hsieh, S.T., Zesch, W., Chan, W.P., Kenny, T.W., Fearing, R., and Full, R.J., 2000 Adhesive force of a single gecko foot-hair. Nature 405, 681-685.

8. Autumn, K., Sitti, M., Liang, Y.A., Peattie, A.M., Hansen, W.R., Sponberg, S., Kenny, T.W., Fearing, R., Israelachvili, J.N., and Full, R.J., 2002 Evidence for van der Waals adhesion in gecko seta. Proc. Natl. Acad. Sci. USA 99, 12252-12256.

9. Yao, H., Gao, H., 2006. Mechanics of robust and releasable adhesion in biology: bottom-up designed hierarchical structures of gecko. J. Mech. Phys. Solids 54, 1120-1146. 
10. Gao, H., Ji, B., Jäger, I.L., Arzt, E., Fratzl., P., 2003. Materials become insensitive to flaws at nanoscale: lessons from nature. Proc. Natl. Acad. Sci. USA 100, 5597-5600.

11. Gao, H., Chen, S., 2005. Flaw tolerance in a thin strip under tension. J. App. Mech. 72, 732-737.

12. Arzt, E., Gorb, S., Spolenak, R., 2003. From micro to nano contacts in biological attachment devices. Proc. Natl. Acad. Sci. USA 100, $10603-$ 10606.

13. Gao, H., Wang, X., Yao, H., Gorb, S., Arzt, E., 2005. Mechanics of hierarchical adhesion structures of geckos. Mechanics of Materials 37, 275-285.

14. Gao, H., Yao, H., 2004. Shape insensitive optimal adhesion of nanoscale fibrillar structures. Proc. Natl. Acad. Sci. USA 101, 7851-7856.

15. H. Yao and H. Gao, 2007. Multi-scale cohesive laws in hierarchical materials, Int. J. Solids Struct., 44, 8177-8193.

16. A.P. Jackson, J.F.V. Vincent and R.M. Turner, 1988. The mechanical design of nacre. Proc. Roy. Soc. Lond. B 234, 415-440.

17. R. Menig, M.H. Meyers, M.A. Meyers and K.S. Vecchio, 2000. Quasistatic and dynamic mechanical response of Haliotis rufescens (abalone) shells. Acta Mat. 48, 2383-2398.

18. R. Menig, M.H.Meyers, M.A. Meyers and K.S. Vecchio, 2001. Quasistatic and dynamic mechanical response of Strombus gigas (conch) shells. Mat. Sci. Eng. A 297, 203-211.

19. W.J. Landis, 1995. The strength of a calcified tissue depends in part on the molecular structure and organization of its constituent mineral crystals in their organic matrix. Bone 16, 533-544.

20. W.J. Landis, K.J. Hodgens, M.J. Song, J. Arena, S. Kiyonaga, M. Marko, C. Owen and B.F. McEwen, 1996. Mineralization of collagen may occur on fibril surfaces: evidence from conventional and high voltage electron microscopy and three dimensional imaging. J. Struct. Biol. 117, 24-35.

21. J.Y. Rho, L. Kuhn-Spearing and P. Zioupos, 1998. Mechanical properties and the hierarchical structure of bone. Med. Eng. \& Phys. 20, 92-102.

22. S. Weiner and H.D. Wagner, 1998. The material bone: structuremechanical function relations. Annual Review of Materials Science 28, 271-298.

23. H. Warshawsky, 1989. Organization of crystals in enamel. Anat. Rec. 224, 242-262.

24. W.Tesch, N. Eidelman, P. Roschger, F. Goldenberg, K. Klaushofer and P. Fratzl, 2001. Graded microstructure and mechanical properties of human crown dentin. Calc. Tissue Int. 69, 147-157.

25. H.D. Jiang, X.Y. Liu, C.T. Lim and C.Y. Hsu, 2005. Ordering of selfassembled nanobiominerals in correlation to mechanical properties of hard tissues. Appl. Phys. Lett. 86, 163901.

26. R.Z. Wang, Z. Suo, A.G. Evans, N. Yao, and I.A. Aksay, 2001. Deformation mechanisms in nacre. J. Mat. Res. 16, 2485-2493.

27. M.J. Buehler, S. Keten, T. Ackbarow, 2008. Theoretical and computational hierarchical nanomechanics of protein materials: Deformation and fracture. Prog. Mat. Sci. 53, 1101-1241.

28. R. Puxkandl, I. Zizak, O. Paris, J. Keckes, W. Tesch, S. Bernstorff, P. Purslow, P. Fratzl, 2001. Viscoelastic properties of collagen: synchrotron radiation investigations and structural model. Phil. Trans. Roy. Soc. Lond. B, 357, 191-197.

29. B. Ji and H. Gao, 2004. Mechanical properties of nanostructure of biological materials. J. Mech. Phys. Solids 52, 1963-1990.

30. B. Chen, P.D. Wu and H. Gao, 2009. A characteristic length for stress transfer in the nanostructure of biological composites. Comp. Sci. Tech. 69, 1160-1164.

31. H.L. Cox, 1952. The elasticity and strength of paper and other fibrous materials. Brit. J. appl. Phys. 3, 72-9.

32. J.W. Hutchinson and H.M. Jensen, 1990. Model of fiber debonding and pullout in brittle composites with friction, Mech. Mater. 9:139-163.

33. J.A. Nairn, 1997. On the use of shear-lag methods for analysis of stress transfer in unidirectional composites, Mech. Mater. 26:63-80.

34. C.L.Tucker III and E. Liang, 1999. Stiffness predictions for unidirectional short fiber composites: review and evaluation, Compos. Sci. Technol. 59:655-671.

35. M.J. Buehler, 2007. Nature designs tough collagen: Explaining the nanostructure of collagen fibrils. Proc. Natl. Acad. Sci. USA 103, 12285 12290.

36. X. Guo and H. Gao, 2005. Bio-inspired material design and optimization. IUTAM Symposium on topological design optimization of structures, machines and materials - status and perspectives, October 26-29, 2005, Rungstedgaard, Copenhagen, Denmark. 